\title{
A Converse Lyapunov Theorem and Robustness with Respect to Unbounded Perturbations for Exponential Dissipativity
}

\author{
Xudong Li and Yuan Guo \\ Department of Mathematics, Lanzhou City University, Lanzhou 730070, China \\ Correspondence should be addressed to Yuan Guo, guomath@hotmail.com \\ Received 18 December 2009; Revised 19 March 2010; Accepted 17 April 2010 \\ Academic Editor: Josef Diblik
}

Copyright (C) 2010 X. Li and Y. Guo. This is an open access article distributed under the Creative Commons Attribution License, which permits unrestricted use, distribution, and reproduction in any medium, provided the original work is properly cited.

This paper is concerned with the nonlinear system $x^{\prime}(t)=f(t, x(t))$. We give a converse Lyapunov theorem and prove robustness of uniform exponential dissipativity with respect to unbounded external perturbations, without assuming $f$ being globally Lipschitz in $x$.

\section{Introduction}

This paper is devoted to the following nonautonomous dynamical system:

$$
x^{\prime}(t)=f(t, x(t)), \quad x(t) \in \mathbb{R}^{n},
$$

where $f: \mathbb{R}^{1} \times \mathbb{R}^{n} \rightarrow \mathbb{R}^{n}$ is always assumed to be a continuous vector field which is locally Lipschitz in space variable $x$. Our main aim is two-fold: one is to give a converse Lyapunov theorem for uniform exponential dissipativity, and the other is to study robustness of uniform exponential dissipativity to unbounded perturbations.

In [1] Lyapunov introduced his famous sufficient conditions for asymptotic stability of (1.1), where we can also find the first contribution to the converse question, known as converse Lyapunov theorems. The answers have proved instrumental, over the years, in establishing robustness of various stability notions and have served as the starting point for many nonlinear control systems design concepts.

Recently Li and Kloeden [2] presented a converse Lyapunov theorem for exponential dissipativity of (1.1) in autonomous case with $f$ being globally Lipschitz in $x$. This result can be seen as a generalization of some classical ones on global exponential asymptotic stability (see, for instance, [3], etc.), and was used by the authors to study robustness of exponential 
dissipativity with respect to small time delays. Here we give a nonautonomous analog of the result; moreover, instead of assuming $f$ to be globally Lipschitz in $x$, we only impose on $f$ the following weaker condition.

(F1) There exists an $L>0$ such that

$$
\langle f(t, x)-f(t, y), x-y\rangle \leq L|x-y|^{2}, \quad \forall x, y \in \mathbb{R}^{n}
$$

where $\langle\cdot, \cdot\rangle$ denotes the inner product in $\mathbb{R}^{n}$.

Note that if $f(t, x)$ is globally Lipschitz in $x$ in a uniform manner with respect to $t \in \mathbb{R}^{1}$, then (F1) is automatically satisfied. However, we emphasize that this condition also allows nonglobally Lipschitz functions. An easy example is the function $f(t, x)=-x^{3}+x$, which is clearly not globally Lipschitz. One observes that

$$
(f(t, x)-f(t, y))(x-y)=-\left(x^{3}-y^{3}\right)(x-y)+(x-y)^{2} \leq|x-y|^{2} .
$$

Then we study robustness property of uniform exponential dissipativity to perturbations. A basic problem in the dynamical theory concerns the robustness of global attractors under perturbations [4]. It is readily known that if a nonlinear system with a global attractor $A$ is perturbed, then the perturbed one also has an attractor $A^{\prime}$ which is near $A$, provided the perturbation is sufficiently small; see, for instance, $[5,6]$, and so forth. However, in general we only know that $A^{\prime}$ is a local attractor. Whether (or under what circumstances) the global feature can be preserved is an interesting but, to the authors' knowledge, still open problem. (For concrete systems there is the hope that one may check the existence of global attractors by using the structure of the systems.) Since the dissipativity of a system usually implies the existence of the global attractor, in many cases the key point to answer the above problem is then reduced to examine the robustness of dissipativity under perturbations.

Such a problem has obvious practical sense. Unfortunately the answer might be negative even if in some simple cases which seem to be very nice at a first glance, as indicated in Example 1.1 below (from which it is seen that dissipativity can be quite sensitive to perturbations).

Example 1.1 (see [7]). Consider the scalar differential equation

$$
x^{\prime}(t)=f(x(t)), \quad \text { where } f(x)=-x e^{-x^{2}} .
$$

It is easy to see that the equilibrium 0 is globally asymptotically stable, and consequently the system is dissipative. However, since for any $\varepsilon>0$ there exists an $r_{0}>0$ such that

$$
f(x)+\varepsilon>0, \quad \forall x>r_{0}
$$

we deduce that any solution $x(t)$ of the perturbed system

$$
x^{\prime}(t)=f(x(t))+\varepsilon
$$

with $x(0)>r_{0}$ goes to $+\infty$ as $t \rightarrow+\infty$. 
Note that $f^{\prime}(x)$ is bounded on $\mathbb{R}^{1}$; hence, $f(x)$ is globally Lipschitz.

In this present work we demonstrate that exponential dissipativity has nice robustness properties. Actually we will show that it is robust under some types of even unbounded perturbations.

This paper is organized as follows. In Section 2 we give a converse Lyapunov theorem mentioned above, and in Section 3 we prove robustness of exponential dissipativity.

\section{A Converse Lyapunov Theorem}

In this section we give a converse Lyapunov theorem which generalizes a recent result in [2]. Let us first recall some basic definitions and facts.

The upper right Dini sup-derivative of a function $y \in C\left((\alpha, \beta), \mathbb{R}^{1}\right)$ is defined as

$$
\frac{d^{+}}{d t} y(t):=\varlimsup_{h \rightarrow 0^{+}} \frac{y(t+h)-y(t)}{h}
$$

Let $I$ be an open interval, and let $U$ be an open subset of $\mathbb{R}^{n}$. Let $V \in C\left(I \times U, \mathbb{R}^{1}\right)$. For $(t, x) \in I \times U$ and $v \in \mathbb{R}^{n}$, define

$$
\mathrm{ND}_{v}^{+} V(t, x):=\varlimsup_{h \rightarrow 0^{+}} \frac{V(t+h, x+h v)-V(t, x)}{h} .
$$

We call $\mathrm{ND}_{v}^{+} V(x)$ the nonautonomous Dini sup-derivative of $V$ at $(t, x)$ along the vector $v$. In case $V$ is differentiable at $(t, x)$, it is easy to see that

$$
\mathrm{ND}_{v}^{+} V(t, x)=\frac{\partial}{\partial t} V(t, x)+\nabla_{x} V(t, x) \cdot v
$$

Lemma 2.1. Let $U$ be an open subset of $\mathbb{R}^{n}$. Assume that the continuous function $V:(\alpha, \beta) \times U \rightarrow$ $\mathbb{R}^{+}$is Lipschitz in $x$ uniformly in $t \in(\alpha, \beta)$, that is, there exists an $L_{V}>0$ such that

$$
|V(t, x)-V(t, y)| \leq L_{V}|x-y|, \quad \forall t \in(\alpha, \beta), x, y \in U,
$$

and let $x(\cdot) \in C^{1}((\alpha, \beta) ; U)$. Then

$$
\frac{d^{+}}{d t} V(t, x(t))=\mathrm{ND}_{g(t)}^{+} V(t, x(t)), \quad \text { where } g(t)=x^{\prime}(t)
$$

Proof. This basic fact is actually contained in [3], and so forth. Here we give a simple proof for the reader's convenience. 
We observe that

$$
\begin{aligned}
V(t+h, x(t+h))-V(t, x(t))= & V(t+h, x(t)+g(t) h+o(h))-V(t, x(t)) \\
= & (V(t+h, x(t)+g(t) h+o(h))-V(t+h, x(t)+g(t) h)) \\
& +(V(t+h, x(t)+g(t) h)-V(t, x(t))) .
\end{aligned}
$$

Since $V(t, x)$ is Lipschitz in $x$, one easily sees that

$$
V(t+h, x(t)+g(t) h+o(h))-V(t+h, x(t)+g(t) h)=o(h) .
$$

Therefore by definition we immediately deduce that

$$
\begin{aligned}
\frac{d^{+}}{d t} V(t, x(t)) & =\varlimsup_{h \rightarrow 0^{+}} \frac{V(t+h, x(t+h))-V(t, x(t))}{h} \\
& =\varlimsup_{h \rightarrow 0^{+}} \frac{V(t+h, x(t)+g(t) h)-V(t, x(t))}{h}=\mathrm{ND}_{g(t)}^{+} V(t, x(t)) .
\end{aligned}
$$

The proof is complete.

We will denote by $\phi(t, \tau)$ the solution operator of $(1.1)$, that is, for each $x \in \mathbb{R}^{n}, x(t):=$ $\phi(t, \tau) x$ is the unique solution of the system with initial value $x(\tau)=x$.

Definition 2.2. System (1.1) is said to be uniformly exponentially dissipative, if there exist positive numbers $B, \lambda$, and $\rho$ such that

$$
|\phi(t, \tau) x| \leq B e^{-\lambda(t-\tau)}|x|+\rho, \quad \forall t \geq \tau, \quad(\tau, x) \in \mathbb{R}^{1} \times \mathbb{R}^{n}
$$

The main result in this section is the following theorem.

Theorem 2.3 (Converse Lyapunov Theorem). Suppose that $f$ satisfies the structure condition (F1). Assume that system (1.1) is uniformly exponentially dissipative.

Then there exists a function $V: \mathbb{R}^{1} \times \mathbb{R}^{n} \rightarrow \mathbb{R}^{+}$satisfying

$$
\begin{gathered}
|x|^{2}-a \leq V(t, x) \leq b|x|^{2}+c \\
|V(t, x)-V(t, y)| \leq L_{V}(|x|+|y|+\rho)|x-y| \\
\operatorname{ND}_{f(t, x)}^{+} V(t, x) \leq-d|x|^{2}+\sigma
\end{gathered}
$$

for all $x, y \in \mathbb{R}^{n}$ and $t \in \mathbb{R}^{1}$, where $a, b, c, d, \sigma$, and $L_{V}$ are appropriate positive constants.

Moreover, if $\rho=0$, namely, the system is uniformly exponentially asymptotically stable, then the constants $a, c$, and $\sigma$ vanish. 
Proof. Since the ODE system (1.1) is exponentially dissipative, there exist positive constants $B, \lambda$, and $\rho$ such that (2.9) holds. Let

$$
T=\frac{1}{\lambda} \ln (2 B)
$$

We first define a function $V_{1}$ as follows: (The techniques used here are adopted from $[2,8]$, etc.)

$$
V_{1}(t, x):=\int_{t}^{t+T}|\phi(s, t) x|^{2} d s, \quad x \in \mathbb{R}^{n}
$$

By (2.9) it is clear that

$$
0 \leq V_{1}(t, x) \leq \int_{t}^{t+T}\left(B e^{-\lambda(s-t)}|x|+\rho\right)^{2} d s \leq \frac{B^{2}}{\lambda}|x|^{2}+2 T \rho^{2}
$$

Let $x(t)=\phi(t, \tau) x, y(t)=\phi(t, \tau) y$, and let $w(t)=x(t)-y(t)$. Then

$$
w^{\prime}(t)=f(t, x(t))-f(t, y(t))
$$

Taking inner product of this equation with $w(t)$, by (F1) one finds that

$$
\frac{1}{2} \frac{d}{d t}|w(t)|^{2} \leq L|w(t)|^{2}
$$

from which it can be easily seen that

$$
|w(t)| \leq|w(\tau)| e^{L(t-\tau)}, \quad t \geq \tau .
$$

Thus we deduce that

$$
|\phi(t, \tau) x-\phi(t, \tau) y| \leq C_{T}|x-y|, \quad \forall x, y \in \mathbb{R}^{n}, t \in[\tau, \tau+T]
$$

where $C_{T}=e^{L T}$. Now for any $x, y \in \mathbb{R}^{n}$ and $t \in \mathbb{R}^{1}$, we have

$$
\begin{aligned}
\left|V_{1}(t, x)-V_{1}(t, y)\right| & =\left|\int_{t}^{t+T}\left(|\phi(s, t) x|^{2}-|\phi(s, t) y|^{2}\right) d s\right| \\
& \leq \int_{t}^{t+T}|\phi(s, t) x-\phi(s, t) y|(|\phi(s, t) x|+|\phi(s, t) y|) d s \\
& \leq C_{T}|x-y| \int_{t}^{t+T}(|\phi(s, t) x|+|\phi(s, t) y|) d s,
\end{aligned}
$$


and it immediately follows by (2.9) that

$$
\left|V_{1}(t, x)-V_{1}(t, y)\right| \leq L_{1}(|x|+|y|+\rho)|x-y|
$$

where $L_{1}$ is independent of $x, y$. This shows that $V_{1}$ satisfies (2.11).

Since

$$
V_{1}(t, \phi(t, \tau) x)=\int_{t}^{t+T}|\phi(s, t) \phi(t, \tau) x|^{2} d s=\int_{t}^{t+T}|\phi(s, \tau) x|^{2} d s
$$

by the choice of $T$ we have that

$$
\begin{aligned}
\frac{d}{d t} V_{1}(t, \phi(t, \tau) x) & =|\phi(t+T, \tau) x|^{2}-|\phi(t, \tau) x|^{2} \\
& =|\phi(t+T, t) \phi(t, \tau) x|^{2}-|\phi(t, \tau) x|^{2} \\
& \leq-|\phi(t, \tau) x|^{2}+\left(B e^{-\lambda T}|\phi(t, \tau) x|+\rho\right)^{2} \\
& \leq-|\phi(t, \tau) x|^{2}+2 B^{2} e^{-2 \lambda T}|\phi(t, \tau) x|^{2}+2 \rho^{2} \\
& \leq-\frac{1}{2}|\phi(t, \tau) x|^{2}+2 \rho^{2} .
\end{aligned}
$$

On the other hand, by Lemma 2.1 we find that

$$
\mathrm{ND}_{f(t, \phi(t, \tau) x)}^{+} V_{1}(t, \phi(t, \tau) x)=\frac{d}{d t} V_{1}(t, \phi(t, \tau) x) \leq-\frac{1}{2}|\phi(t, \tau) x|^{2}+2 \rho^{2}
$$

Setting $t=\tau$, one obtains that

$$
\mathrm{ND}_{f(\tau, x)}^{+} V_{1}(\tau, x) \leq-\frac{1}{2}|x|^{2}+2 \rho^{2}
$$

which indicates that $V_{1}$ satisfies (2.12).

Now let us define another Lyapunov function $V_{2}$. For this purpose we take a nonnegative function $\alpha(s)$ as

$$
\alpha(s)=\max \left(s^{2}-\rho_{0}^{2}, 0\right), \quad s \geq 0,
$$

where $\rho_{0}=(2 B+1) \rho$. Then

$$
|\alpha(s)-\alpha(r)| \leq(s+r)|s-r|, \quad \forall s, r \geq 0 .
$$


Indeed, if $s, r<\rho_{0}$, then the estimate clearly holds true. So we may assume without loss of generality that $s \geq r$ with $s \geq \rho_{0}$. We have

$$
|\alpha(s)-\alpha(r)|=\alpha(s)-\alpha(r)=\left(s^{2}-\rho_{0}^{2}\right)-\alpha(r) \leq\left(s^{2}-\rho_{0}^{2}\right)-\left(r^{2}-\rho_{0}^{2}\right)=(s+r)|s-r| .
$$

Let

$$
V_{2}(t, x)=\sup _{s \geq t} \alpha(|\phi(s, t) x|), \quad(t, x) \in \mathbb{R}^{1} \times \mathbb{R}^{n}
$$

We claim that we actually have

$$
V_{2}(t, x)=\sup _{t \leq s \leq t+T} \alpha(|\phi(s, t) x|)
$$

Indeed, if $|x| \leq 2 \rho$, then by (2.9) we deduce that $|\phi(s, t) x| \leq \rho_{0}$ for all $s \geq t$. On the other hand, by the definition of $\alpha(r)$ we have $\alpha(r)=0$ for all $0 \leq r \leq \rho_{0}$. Therefore in case $|x| \leq 2 \rho$, one trivially has

$$
V_{2}(t, x)=0=\sup _{t \leq s \leq t+T} \alpha(|\phi(s, t) x|)
$$

Now assume that $|x|>2 \rho$. Then by the choice of $T$ we find that

$$
|\phi(s, t) x| \leq B e^{-\lambda T}|x|+\rho \leq \frac{1}{2}|x|+\rho \leq|x|, \quad s \geq t+T .
$$

Since $\phi(t, t) x=x$ and $\alpha(s)$ is nondecreasing in $s$, one immediately deduces that

$$
V_{2}(t, x)=\sup _{t \leq s \leq t+T} \alpha(|\phi(s, t) x|)
$$

which completes the proof of (2.30).

By (2.9), (2.19), and (2.27) we have

$$
\begin{aligned}
V_{2}(t, x) & =\sup _{t \leq s \leq t+T} \alpha(|\phi(s, t) x|)=\sup _{t \leq s \leq t+T}((\alpha(|\phi(s, t) x|)-\alpha(|\phi(s, t) y|))+\alpha(|\phi(s, t) y|)) \\
& \leq \sup _{t \leq s \leq t+T}(\alpha(|\phi(s, t) x|)-\alpha(|\phi(s, t) y|))+V_{2}(t, y) \\
& \leq C_{T}(B|x|+B|y|+2 \rho)|x-y|+V_{2}(t, y) .
\end{aligned}
$$

Therefore

$$
V_{2}(t, x)-V_{2}(t, y) \leq C_{T}(B|x|+B|y|+2 \rho)|x-y| .
$$


Since $x$ and $y$ are arbitrary, we conclude that

$$
\left|V_{2}(t, x)-V_{2}(t, y)\right| \leq C_{T}(B|x|+B|y|+2 \rho)|x-y|, \quad \forall t \in \mathbb{R}^{1}, x, y \in \mathbb{R}^{n} .
$$

By the definition of $\alpha(s)$ it is clear that

$$
s^{2}-\rho_{0}^{2} \leq \alpha(s) \leq s^{2}, \quad \forall s \geq 0 .
$$

It immediately follows that

$$
V_{2}(t, x)=\sup _{s \geq t} \alpha(|\phi(s, t) x|) \geq \alpha(|\phi(t, t) x|)=\alpha(|x|) \geq|x|^{2}-\rho_{0}^{2} .
$$

We also infer from (2.9) that

$$
|\phi(s, t) x| \leq B e^{-\lambda(s-t)}|x|+\rho \leq B|x|+\rho, \quad \forall s \geq t .
$$

Therefore by definition of $V_{2}$ and the monotonicity property of $\alpha(s)$, we have

$$
V_{2}(t, x) \leq \alpha(B|x|+\rho) \leq(B|x|+\rho)^{2} \leq 2 B^{2}|x|^{2}+2 \rho^{2} .
$$

In conclusion we have

$$
|x|^{2}-\rho_{0}^{2} \leq V_{2}(t, x) \leq 2 B^{2}|x|^{2}+2 \rho^{2}, \quad \forall(t, x) \in \mathbb{R}^{1} \times \mathbb{R}^{n} .
$$

Note that if $t>\tau$, then

$$
\begin{aligned}
V_{2}(t, \phi(t, \tau) x) & =\sup _{s \geq t} \alpha(|\phi(s, t) \phi(t, \tau) x|)=\sup _{s \geq t} \alpha(|\phi(s, \tau) x|) \\
& \leq \sup _{s \geq \tau} \alpha(|\phi(s, \tau) x|)=V_{2}(\tau, x) .
\end{aligned}
$$

This implies that $V_{2}(t, \phi(t, \tau) x)$ is nonincreasing in $t$.

Now set

$$
V(t, x)=V_{1}(t, x)+V_{2}(t, x), \quad \forall(t, x) \in \mathbb{R}^{1} \times \mathbb{R}^{n} .
$$

Invoking (2.15), (2.21), and (2.25), we find that $V$ is a Lyapunov function satisfying all the required properties in the theorem.

In case $\rho=0$, it can be easily seen from the above argument that $a, c, \sigma=0$.

The proof is complete.

Remark 2.4. If we assume that $f(t, x)$ is also locally Lipschitz in $t$, then $\phi(t, \tau) x$ is locally Lipschitz in $\tau$ as well. Now assume that $y(t)$ is locally Lipschitz in $t$. Then by the construction of $V_{1}$ and $V_{2}$ one easily verifies that $V(t, y(t))$ is locally Lipschitz in $t$. Consequently $V(t, y(t))$ has derivative in $t$ almost everywhere. 


\section{Robustness of Exponential Dissipativity to Perturbations}

As for the applications of the converse Lyapunov theorem given in Section 2, we consider in this section the robustness of exponential dissipativity to perturbations.

\subsection{Robustness to External Perturbations}

Consider the following perturbed system:

$$
x^{\prime}(t)=f(t, x(t))+p(t, x(t)), \quad x(t) \in \mathbb{R}^{n},
$$

where $p(t, x)$ is a continuous function which corresponds to external perturbations.

Denote by $P_{\varepsilon}$ the family of continuous functions $p(t, x)$ that satisfies the following growth condition:

$$
|p(t, x)| \leq \varepsilon|x|+w(t), \quad \forall t, x \in \mathbb{R}^{1},
$$

where $w$ is a continuous nonnegative function on $\mathbb{R}^{1}$ with

$$
\int_{t}^{t+1} w^{2}(s) d s \leq A^{2}, \quad \forall t \in \mathbb{R}^{1}
$$

for some $A>0$. Our main result in this part is contained in the following theorem.

Theorem 3.1. Assume that $f(t, x)$ is locally Lipschitz in $(t, x)$ and satisfies (F1). Suppose that the system (1.1) is uniformly exponentially dissipative.

Then there exists an $\varepsilon>0$ sufficiently small such that, for any $p \in P_{\varepsilon}$, the perturbed system (3.1) is uniformly exponentially dissipative.

Remark 3.2. Suppose that $p(t, x)$ satisfies a sublinear growth condition

$$
|p(t, x)| \leq c_{0}|x|^{\theta}+w(t)
$$

where $0 \leq \theta<1$, and $w$ is as in (3.3). Then one easily verifies that, for any $\varepsilon>0$, there exists a $C_{\varepsilon}>0$ such that

$$
|p(t, x)| \leq \varepsilon|x|+w(t)+C_{\varepsilon}, \quad \forall(t, x) \in \mathbb{R}^{1} \times \mathbb{R}^{n},
$$

namely, $p \in P_{\varepsilon}$. Hence the conclusion of the theorem naturally holds.

Proof of Theorem 3.1. Let $V$ be the Lyapunov function of the unperturbed system given in Theorem 2.3, and take $\varepsilon=d /\left(4 L_{V}\right)$, where $d$ and $L_{V}$ are the constants in (2.11) and (2.12). We show that for any $p \in P_{\varepsilon}$ the perturbed system (3.1) is uniformly exponentially dissipative.

For simplicity in writing we set

$$
g(t, x)=f(t, x)+p(t, x)
$$


Let $x=x(t)$ be any solution of the perturbed system (3.1) with initial value $x(\tau)=x_{0}$. By Remark 2.4 we know that $V(t, x):=V(t, x(t))$ is locally Lipschitz in $t$ and hence has derivative almost everywhere. Note that at any point $t$ where $V(t, x)$ has derivative we necessarily have

$$
\frac{d^{+}}{d t} V(t, x)=\frac{d}{d t} V(t, x)
$$

Now by Lemma 2.1 we find that

$$
\begin{aligned}
\frac{d^{+}}{d t} V(t, x)= & \varlimsup_{h \rightarrow 0^{+}} \frac{1}{h}(V(t+h, x+h g(t, x))-V(t, x)) \\
\leq & \varlimsup_{h \rightarrow 0^{+}} \frac{1}{h}(V(t+h, x+h g(t, x))-V(t+h, x+h f(t, x))) \\
& +\varlimsup_{h \rightarrow 0^{+}} \frac{1}{h}(V(t+h, x+h f(t, x))-V(t, x)) \\
= & \varlimsup_{h \rightarrow 0^{+}} \frac{1}{h}(V(t+h, x+h g(t, x))-V(t+h, x+h f(t, x)))+\mathrm{ND}_{f(t, x)}^{+} V(t, x) .
\end{aligned}
$$

By (2.11) we have

$$
\begin{aligned}
& |V(t+h, x+h g(t, x))-V(t+h, x+h f(t, x))| \\
& \quad \leq h L_{V}(|x+h g(t, x)|+|x+h f(t, x)|+\rho)|p(t, x)| .
\end{aligned}
$$

Therefore

$$
\begin{aligned}
\frac{d^{+}}{d t} V(t, x) & \leq \mathrm{ND}_{f(t, x)}^{+} V(t, x)+L_{V}(2|x|+\rho)|p(t, x)| \\
& \leq-d|x|^{2}+\sigma+L_{V}(2|x|+\rho)(\varepsilon|x|+w(t))
\end{aligned}
$$

Since $\varepsilon \leq d /\left(4 L_{V}\right)$, we find that

$$
\begin{aligned}
-d & |x|^{2}+\sigma+L_{V}(2|x|+\rho)(\varepsilon|x|+w(t)) \\
& =-d|x|^{2}+\sigma+2 L_{V} \varepsilon|x|^{2}+\left(L_{V} \rho \varepsilon+2 L_{V} w(t)\right)|x|+L_{V} \rho w(t) \\
& \leq-\frac{d}{2}|x|^{2}+\sigma+\left(\frac{d}{4} \rho+2 L_{V} w(t)\right)|x|+L_{V} \rho w(t) \\
& \leq-\frac{d}{4}|x|^{2}+c_{1}|w(t)|^{2}+c_{2}
\end{aligned}
$$

where $c_{1}, c_{2}>0$ are appropriate numbers (which are independent of the initial values). Thus

$$
\frac{d^{+}}{d t} V(t, x(t)) \leq-\frac{d}{4}|x(t)|^{2}+c_{1}|w(t)|^{2}+c_{2} \leq(\operatorname{by}(2.10)) \leq-\Lambda V(t, x(t))+c_{1}|w(t)|^{2}+c_{3}
$$


where

$$
\Lambda=\frac{d}{4 b}, \quad c_{3}=c_{2}+\frac{d c}{4 b}
$$

and $b$ and $c$ are the constants in (2.10). In particular, we have

$$
\frac{d^{+}}{d t} V(t, x(t)) \leq c_{1}|w(t)|^{2}+c_{3}, \quad t \geq \tau
$$

and it follows that

$$
V(t, x) \leq V\left(\tau, x_{0}\right)+c_{1} \int_{\tau}^{t}|w(s)|^{2} d s+c_{3}(t-\tau) \leq b\left|x_{0}\right|^{2}+c_{4}
$$

for $t \in[\tau, \tau+1]$, where $c_{4}=c+c_{1} A^{2}+c_{3}$.

Integrating both sides of (3.12) from $t$ to $t+1$, one finds that

$$
\int_{t}^{t+1} \frac{d^{+}}{d s} V(s, x(s)) d s \leq-\Lambda \int_{t}^{t+1} V(s, x(s)) d s+c_{1} A^{2}+c_{3} .
$$

Since $V(t, x(t))$ is locally Lipschitz in $t$, we find that

$$
V(t+1, x(t+1))-V(t, x(t))=\int_{t}^{t+1} \frac{d}{d s} V(s, x(s)) d s=\int_{t}^{t+1} \frac{d^{+}}{d s} V(s, x(s)) d s
$$

Hence

$$
\begin{aligned}
\frac{d}{d t} \int_{t}^{t+1} V(s, x(s)) d s & =V(t+1, x(t+1))-V(t, x(t))=\int_{t}^{t+1} \frac{d^{+}}{d s} V(s, x(s)) d s \\
& \leq-\Lambda \int_{t}^{t+1} V(s, x(s)) d s+c_{5}
\end{aligned}
$$

where $c_{5}=c_{1} A^{2}+c_{3}$. By the classical Gronwall lemma and (3.15) we obtain

$$
\begin{aligned}
\int_{t}^{t+1} V(s, x(s)) d s & \leq \int_{\tau}^{\tau+1} V(s, x(s)) d s e^{-\Lambda(t-\tau)}+\frac{c_{5}}{\Lambda} \\
& \leq b\left|x_{0}\right|^{2} e^{-\Lambda(t-\tau)}+\left(c+\frac{c_{5}}{\Lambda}\right), \quad t \geq \tau .
\end{aligned}
$$

Now for any fixed $s \in[t, t+1]$ we integrate (3.14) from $s$ to $t+1$ and find that

$$
V(t+1, x(t+1)) \leq V(s, x(s))+c_{1} A^{2}+c_{3} .
$$


Further integrating the above inequality in $s$ from $t$ to $t+1$, it yields

$$
V(t+1, x(t+1)) \leq \int_{t}^{t+1} V(s, x(s)) d s+c_{1} A^{2}+c_{3} \leq b\left|x_{0}\right|^{2} e^{-\Lambda(t-\tau)}+c_{6}, \quad t \geq \tau,
$$

where $c_{6}=\left(c+c_{5} / \Lambda\right)+c_{1} A^{2}+c_{3}$. By (2.10) one concludes that

$$
|x(t)|^{2} \leq M e^{-\Lambda(t-\tau)}\left|x_{0}\right|^{2}+c_{7}, \quad t \geq \tau+1,
$$

where $M=b e^{\Lambda}$, and $c_{7}=c_{6}+a$.

We also deduce by (2.10) and (3.15) that

$$
|x(t)|^{2} \leq b\left|x_{0}\right|^{2}+c_{4}+a \leq M e^{-\Lambda(t-\tau)}\left|x_{0}\right|^{2}+c_{4}+a, \quad t \in[\tau, \tau+1] .
$$

Therefore, (3.22) and (3.23) complete the proof of what we desired.

As a direct consequence of Theorem 3.1, we have the following interesting result.

Corollary 3.3. Assume that $f$ satisfies (F1) and the following sublinear growth condition

$$
|f(t, x)| \leq c_{0}|x|^{\theta}+w(t)
$$

where $0 \leq \theta<1$, and $w$ is as in $(P 1)$.

Then system (1.1) is necessarily not uniformly exponentially dissipative.

Proof. Suppose that (1.1) is uniformly exponentially dissipative. Then by Theorem 3.1, the perturbed system (3.1) is uniformly exponentially dissipative for any perturbation $p(t, x)$, provided $(P 1)$ is satisfied with $\varepsilon>0$ sufficiently small. On the other hand, taking $p(t, x)=\varepsilon x$ for any $\varepsilon>0$, by sublinear growth condition on $f$ one easily examine by using standard argument that the perturbed system

$$
x^{\prime}(t)=\varepsilon x(t)+f(t, x(t))
$$

is not dissipative. This leads to a contradiction and proves the conclusion.

\subsection{The Cohen-Grossberg Neural Networks with Unbounded External Inputs and Disturbances}

As another simple example of the application of Theorem 2.3, we consider the following Cohen-Grossberg neural networks with variable coefficients and multiple delays considered recently in [9]:

$$
x_{i}^{\prime}(t)=-d_{i}\left(x_{i}\right)\left(c_{i}\left(x_{i}\right)-\sum_{j=1}^{n} a_{i j}(t) f_{j}\left(x_{j}\right)-\sum_{j=1}^{n} a_{i j}(t) f_{j}\left(x_{j}\left(t-\tau_{j}(t)\right)\right)+w_{i}(t, x)\right),
$$


where $w_{i}(t, x)$ denote outside inputs and disturbances, $x=\left(x_{1}, \ldots, x_{n}\right)$, and

$$
\tau_{i} \in C\left(\mathbb{R}^{1} ;[0, \eta]\right), \quad i=1,2, \ldots, n
$$

denote time delays, where $0<\eta<\infty$. For the physical meaning of the coefficients we refer the reader to [9], and so forth. In case $w_{i}$ is bounded and independent of $x$, the exponential dissipativity is actually considered in [9]. Here we discuss the more general case. As in [9] we assume that

(H1) $d_{i}(s)$ are bounded and locally Lipschitz,

(H2) each function $g_{i}(s)=d_{i}(s) c_{i}(s)$ belongs to $C^{1}$; moreover,

$$
g_{i}^{\prime}(s) \geq m_{i}>0, \quad \forall s \in \mathbb{R}^{1}
$$

(H3) $a_{i j}(t), b_{i j}(t)$, and $f_{j}(s)$ are bounded continuous functions.

Theorem 3.4. Assume (H1)-(H3). Then there exists an $\varepsilon>0$ sufficiently small such that for any continuous functions $w_{i}(t, x)$ satisfying

$$
\left|w_{i}(t, x)\right| \leq \varepsilon|x|+w(t), \quad(t, x) \in \mathbb{R}^{1} \times \mathbb{R}^{n}, 1 \leq i \leq n,
$$

where $w(t)$ is a function as in (3.3), system (3.26) is uniformly exponentially dissipative.

Proof. Consider the system

$$
x^{\prime}(t)=f(x), \quad \text { where } f(x)=-\left(g_{1}\left(x_{1}\right), \ldots, g_{n}\left(x_{n}\right)\right)
$$

with $g_{i}(s)=d_{i}(s) c_{i}(s)$. By (H2) one easily verifies that $f(x)$ satisfies (F1); moreover, system (3.30) is exponentially dissipative. Let $V$ be the Lyapunov function of the system given by Theorem 2.3. We show that if $\varepsilon>0$ is sufficiently small, then (3.26) is uniformly exponentially dissipative, provided (3.29) is fulfilled.

For simplicity we write

$$
p(t, x, y)=\left(p_{1}(t, x, y), \ldots, p_{n}(t, x, y)\right), \quad t \in \mathbb{R}^{1}, x, y \in \mathbb{R}^{n},
$$

where

$$
p_{i}(t, x, y)=d_{i}\left(x_{i}\right)\left(\sum_{j=1}^{n} a_{i j}(t) f_{j}\left(x_{j}\right)+\sum_{j=1}^{n} a_{i j}(t) f_{j}\left(y_{j}\right)-w_{i}(t, x)\right)
$$

Then system (3.26) can be reformulated as

$$
x^{\prime}(t)=f(x(t))+p\left(t, x(t), x_{t}\right),
$$


where

$$
x_{t}=\left(x_{1}\left(t-\tau_{1}(t)\right), \ldots, x_{n}\left(t-\tau_{n}(t)\right)\right) .
$$

We observe by (H1), (H3), and (3.29) that

$$
\left|p_{i}(t, x, y)\right| \leq D_{i}\left(M_{i}+\left|w_{i}(t, x)\right|\right) \leq D_{i} \varepsilon|x|+D_{i}\left(w(t)+M_{i}\right)
$$

where $D_{i}=\sup _{s \in \mathbb{R}^{1}}\left|d_{i}(s)\right|$, and

$$
M_{i}=\sup _{t \in \mathbb{R}^{1}, x, y \in \mathbb{R}^{n}}\left(\sum_{j=1}^{n}\left|a_{i j}(t) f_{j}\left(x_{j}\right)\right|+\sum_{j=1}^{n}\left|a_{i j}(t) f_{j}\left(y_{j}\right)\right|\right) .
$$

Therefore

$$
\begin{aligned}
|p(t, x, y)| & =\left(\left|p_{1}(t, x, y)\right|^{2}+\cdots+\left|p_{n}(t, x, y)\right|^{2}\right)^{1 / 2} \leq K\left(\left|p_{1}(t, x, y)\right|+\cdots+\left|p_{n}(t, x, y)\right|\right) \\
& \leq(K D) \varepsilon|x|+\left(D w(t)+\sum_{i=1}^{n} D_{i} M_{i}\right):=(K D) \varepsilon|x|+\tilde{w}(t)
\end{aligned}
$$

where $K>0$ is a constant which only depends on the dimension $n$ of the phase space $\mathbb{R}^{n}$, and $D=D_{1}+\cdots+D_{n}$. Note that the function

$$
\tilde{w}(t)=D w(t)+\sum_{i=1}^{n} D_{i} M_{i}
$$

satisfies (3.3) with $A$ therein replaced by another appropriate constant $\tilde{A}>0$.

Now assume that $(K D) \varepsilon \leq d /\left(4 L_{V}\right)$, where $d$ and $L_{V}$ are the constants in (2.11) and (2.12). By repeating the same argument as in the proof of Theorem 3.1 with almost no modification, one can show that there exist constants $M, \Lambda$ and $c>0$ such that for any solution $x(t)$ of system (3.26) with initial value

$$
x(\tau+s)=\psi(s), \quad s \in[-\eta, 0],
$$

where $\psi \in C\left([-\eta, 0] ; \mathbb{R}^{n}\right)$, we have

$$
|x(t)|^{2} \leq M e^{-\Lambda(t-\tau)}|x(\tau)|^{2}+c \leq M e^{-\Lambda(t-\tau)}\|\psi\|^{2}+c, \quad t \geq \tau .
$$

Here $\|\psi\|$ denotes the usual norm of $\psi$ in $C\left([-\eta, 0] ; \mathbb{R}^{n}\right)$. We omit the details.

The proof of the theorem is complete.

Remark 3.5. The above result contains Theorem 3.1 in [9] as a particular case. 


\section{Acknowledgments}

The authors highly appreciate the work of the anonymous referees whose comments and suggestions helped them greatly improve the quality of the paper in many aspects. This paper is supported by NNSF of China (10771159).

\section{References}

[1] A. M. Lyapunov, "The general problem of the stability of motion," International Journal of Control, vol. 55, no. 3, pp. 531-534, 1992 (Russian).

[2] D. Li and P. E. Kloeden, "Robustness of asymptotic stability to small time delays," Discrete and Continuous Dynamical Systems. Series A, vol. 13, no. 4, pp. 1007-1034, 2005.

[3] T. Yoshizawa, Stability Theory by Lyapunov's Second Method, The Mathematical Society of Japan, Tokyo, Japan, 1996.

[4] J. K. Hale, Asymptotic Behavior of Dissipative Systems, vol. 25 of Mathematical Surveys and Monographs, American Mathematical Society, Providence, RI, USA, 1988.

[5] D. Li, "Morse decompositions for general dynamical systems and differential inclusions with applications to control systems," SIAM Journal on Control and Optimization, vol. 46, no. 1, pp. 35-60, 2007.

[6] J. C. Robinson, Infinite-Dimensional Dynamical Systems, Cambridge Texts in Applied Mathematics, Cambridge University Press, Cambridge, UK, 2001.

[7] P. E. Kloeden and S. Siegmund, "Bifurcations and continuous transitions of attractors in autonomous and nonautonomous systems," International Journal of Bifurcation and Chaos, vol. 15, no. 3, pp. 743-762, 2005.

[8] S. F. Hafstein, "A constructive converse Lyapunov theorem on exponential stability," Discrete and Continuous Dynamical Systems. Series A, vol. 10, no. 3, pp. 657-678, 2004.

[9] M. Jiang, Y. Shen, and X. Liao, "Boundedness and global exponential stability for generalized CohenGrossberg neural networks with variable delay," Applied Mathematics and Computation, vol. 172, no. 1, pp. 379-393, 2006. 